\title{
Reconstruction of full thickness wounds using glyaderm in a single-staged procedure
}

\author{
Melissa de Henau • Anne Sophie Kruit $\mathbb{D} \cdot$ Dietmar J. O. Ulrich
}

Received: 6 April 2020/ Accepted: 2 February 2021 / Published online: 23 February 2021

(C) The Author(s) 2021

\begin{abstract}
Introduction In large full-thickness skin defects, donor site morbidity limits the available thickness and surface of skin autografts and therefore only splitthickness skin grafts are possible for reconstruction. Dermal equivalents can be added to these splitthickness grafts to acquire an anatomically better skin reconstruction. Glyaderm is a human derived, acellular dermis and up until now has only been used in a two-staged procedure. This report describes results of a case series using Glyaderm and split-thickness skin grafts in a single-staged procedure.

Methods Glyaderm was introduced in 2017 in Radboudumc (Nijmegen, The Netherlands). Glyaderm and autologous split-skin grafts were simultaneously applied to the wounds. In cases with large wound surfaces or wounds covering highly mobile areas, negative pressure wound therapy was additionally applied. The first ten cases were followed with regular intervals post-operatively, assessing graft take, scar
\end{abstract}

Melissa de Henau and Anne Sophie Kruit have a shared first co-authorship.

A project collaboration between Radboudumc and ETBBISLIFE.

M. de Henau · A. S. Kruit $(\bowtie)$ · D. J. O. Ulrich Department of Plastic and Reconstructive Surgery, Radboud University Medical Center, Geert Grooteplein Zuid 10, 6523 GA Nijmegen, The Netherlands

e-mail: annesophie.kruit@radboudumc.nl appearance, post-operative wound problems and reinterventions.

Results Patients were aged 3 weeks to 76 years-old. Treated skin surface varied from $1-16 \%$ total body surface. Wounds resulted from trauma $(\mathrm{n}=4)$, burns $(n=4)$ or soft tissue infections $(n=2)$. Follow-up varied from 4 months to 1.5 years. No complications occurred after surgery. Average take rate was $98 \%$. Two patients had a later re-intervention to further improve the aesthetic appearance of the scarred area. Conclusion Our first results with the application of Glyaderm in a single-staged procedure provided good healing, graft take and scar appearance. Glyaderm was found a suitable dermal substitute in the treatment of full thickness wounds.

Keywords Full thickness wound - Dermal substitute · Glyaderm - Skin graft - Negative pressure wound therapy

\section{Introduction}

One of the main treatment options for skin defects is split-thickness skin grafting (STSG). However, when the surface and depth of the skin defect is extensive, such as in severely burned patients, donor site morbidity limits the available thickness and surface of skin autografts. This necessitates STSG with 
meshing and expansion of the grafts, resulting in unaesthetic healing and may lead to hypertrophic scars.

In the past two decades, the concept of a bi-layered wound coverage in the treatment of extensive full thickness wounds has become widely accepted (Demling et al. 2007; Nguyen et al. 2010). In this concept, a dermal substitute is used in combination with a STSG. The combined use of a dermal and epidermal analog mimics normal skin anatomy and may therefore improve aesthetic outcome and diminish scar hypertrophy (Pirayesh et al. 2007).

A dermal substitute can be defined as a bio-matrix that fulfills the functions of the normal cutaneous dermal layer. The general requirements of a dermal substitute are protecting the wound from infection and fluid loss and providing a stable and biodegradable template that improves the synthesis of new dermal tissue. The dermal substitute thus provides a scaffold during the healing process to the cells infiltrating the wound bed to promote tissue growth and to enhance wound healing (Lee 2000). This results in newly formed dermal tissue rather than scar tissue which is better able to resist tear forces and is more elastic and thus less painful (van der Veen et al. 2010).

Several dermal substitutes have become available, derived from synthetic or biological materials. The biological materials can either be derived from allogeneic material (human) or xenogeneic material (mainly porcine and bovine). Biological dermal substitutes show high similarity to native dermis and provide a 3-dimensional extra-cellular matrix of collagen and elastin without cells (Shahrokhi et al. 2014). To date, the biological derivates are preferred in clinical practice (Truong et al. 2005; Shahrokhi et al. 2014).

The use of the first human-derived dermal substitute, AlloDerm ${ }^{\circledR}$ was described in 1995 (Wainwright 1995). AlloDerm ${ }^{\circledR}$ is made of human cadaver skin that has been chemically treated in multiple stages to remove all donor cells. Good results have been described on its use, however, the production of AlloDerm ${ }^{\circledR}$ is a complex process and comes at high costs (Reported price in 2013; $€ 21.7 / \mathrm{cm}^{2}$; Butterfield 2013).

In 2008, Richters et al. developed a cost-efficient technique to create an acellular dermal matrix from glycerol preserved allogeneic skin (Richters et al. 2008). The resulting non-profit dermal substitute is available as Glyaderm (Glycerol preserved Acellular Dermis) by the Euro Tissue Bank (ETB-BISLIFE, Skin Department, Beverwijk, The Netherlands). The use of glycerol preservation and $\mathrm{Na}-\mathrm{OH}$ incubation removes all antigenetic structures and cells.

To date, treatment of full thickness defects with Glyaderm has only been described as a two-staged procedure. In the first stage, Glyaderm is applied to a granulating wound bed and covered by sterile dressings. The second stage, split thickness autografting, is usually performed 5-7 days later (Pirayesh et al. 2015). This protocol has shown to have good clinical outcomes, with better scar quality and aesthetic outcomes compared to STSG alone. The main disadvantage of this protocol is the use of two stages. Glyaderm was introduced in 2017 in the Radboudumc (Nijmegen, the Netherlands) to aid closure of selected full thickness wounds, for instance wounds overlying a joint surface or with a large surface. A new protocol was used, were Glyaderm was applied in a singlestaged procedure. This article describes the results of the first ten cases.

\section{Materials and methods}

Ten patients who presented in the Radboud University Medical Center between 2017 and 2019 with full thickness defects of various origins were treated with Glyaderm and STSG in a single-stage procedure (Table 1). When a healthy wound bed was reached after debriding, the wounds were covered in one session with Glyaderm (average thickness $0.3 \mathrm{~mm}$ ) and STSG from various donor sites, depending on the localization of the wound. Following the application and fixation of Glyaderm to cover the entire wound, the STSG was applied on top of it. Both layers were fixated using absorbable sutures. In seven cases with large wound surfaces or in wounds spanning highly mobile areas, negative pressure wound therapy (NPWT) was applied immediately after wound coverage intra-operatively. The NPWT was used continuously at a pressure of $-125 \mathrm{mmHg}$ until the first wound inspection at 5 days post-operative. After removal of the NPWT device, wounds were covered with paraffin gauzes until a stable skin ingrowth was reached. In the three remaining cases where no NPWT was applied, wounds were covered with paraffin gauzes and a tie-over until the first wound inspection. 
Table 1 Patient characteristics and outcomes

\begin{tabular}{|c|c|c|c|c|c|c|c|c|}
\hline $\begin{array}{l}\mathrm{Pt} \\
\text { no }\end{array}$ & $\begin{array}{l}\text { Age } \\
\text { (years) }\end{array}$ & Gender & $\begin{array}{l}\text { Date of } \\
\text { surgery }\end{array}$ & Defect area & Defect cause & $\begin{array}{l}\text { TBSA } \\
(\%)\end{array}$ & $\begin{array}{l}\text { Take rate } \\
(\%)\end{array}$ & $\begin{array}{l}\text { NPWT } \\
\text { (days) }\end{array}$ \\
\hline 1 & 19 & Female & 2017-03-03 & Left flank & Infection & 16 & 96 & 5 \\
\hline 2 & 28 & Female & 2017-08-02 & $\begin{array}{l}\text { Amputation stump left } \\
\text { upper leg }\end{array}$ & Trauma & 6 & 98 & 5 \\
\hline 3 & 77 & Female & 2017-08-07 & Right lower leg & Grade 3 burn & 3 & 100 & - \\
\hline 4 & 22 & Male & 2017-08-08 & Left dorsal wrist & Trauma & 1 & 99 & - \\
\hline 5 & 25 & Male & 2018-02-12 & Lower back & Grade $2-3$ burn & 11 & 99 & 5 \\
\hline 6 & 26 & Male & 2018-06-12 & Thorax & Grade $2-3$ burn & 6 & 99 & - \\
\hline 7 & 76 & Male & 2018-10-31 & Right leg & Trauma & 16 & 96 & 5 \\
\hline 8 & 6 & Male & 2018-10-20 & Left knee region & $\begin{array}{l}\text { Deep grade } 2 \\
\text { burn }\end{array}$ & 2 & 100 & 5 \\
\hline 9 & 3 week & Female & 2019-05-27 & Lower back & Trauma & 7 & 90 & 5 \\
\hline 10 & 3 week & Female & 2019-06-24 & $\begin{array}{l}\text { Buttocks and dorsal upper } \\
\text { legs }\end{array}$ & Infection & 10 & 98 & 5 \\
\hline
\end{tabular}

$T B S A$ total body surface area, $N P W T$ negative pressure wound therapy

Graft take rate was evaluated at 5-7 days, 2 months and every 6 months thereafter by clinical evaluation. Scar aesthetic appearance and hypertrophy were evaluated and recorded by the threating physician.

\section{Case presentation}

Case 1

Our first case was a 19-year-old healthy girl who suffered necrotizing fasciitis in February 2017. This resulted from a complicated tonsillitis with left-sided pleural empyema and thorax drainage. The necrotizing fasciitis developed around the drain insertion. The skin and fascia of her left flank were excised in multiple sessions to reach a healthy wound bed. This resulted in a wound with an affected total body surface area (TBSA) of $12 \%\left(900 \mathrm{~cm}^{2}\right)$ (Fig. 1). One week after the initial debridement, the wound was covered in one session with Glyaderm and STSG taken from both upper legs. A negative pressure wound therapy (NPWT) device was applied over the grafts to aid in ingrowth and was left in place for 5 days. The graft take rate was $96 \%$. After 6 months, she developed an elastic skin and the mesh pattern faded nicely. She had a slight tight feeling at her wound and there was a small hypertrophic area at the cranial side of the wound. At 1.5 year, the aesthetic appearance of the wound further increased with natural color and smoother transition of scar to native skin at the wound edges. Nevertheless, her scar is currently being reconstructed in multiple sessions by using tissue expansion, aiding in coverage of the scarred area with native skin.

Case 2

A 76-year-old healthy male was brought in with a severe deglovement injury of his entire right leg in a low-speed accident with a truck (TBSA 16\%) (Fig. 2). He had multiple fractures of his fibular head, patella and distal tibia, without neurovascular damage. He was immediately taken for surgery, with removal of necrotic skin and fasciotomy. At a second and third look, additional necrotectomies were performed and a NTWP device was placed. Three weeks later there was a stable situation and the defect was ready for coverage. A local hemisoleus muscle flap was used to cover a deep defect at the proximal tibial area, followed by Glyaderm + STSG coverage of the entire leg in a single procedure. The STSG donor sites were the left leg and abdomen. Average take after 2 weeks was $98.5 \%$. At 5 months postoperatively, the wound was fully closed and the mesh pattern started to fade. There was a tightness around the knee that restricted knee flexion to 60 degrees, for which an intensive physical therapy program was followed until a 


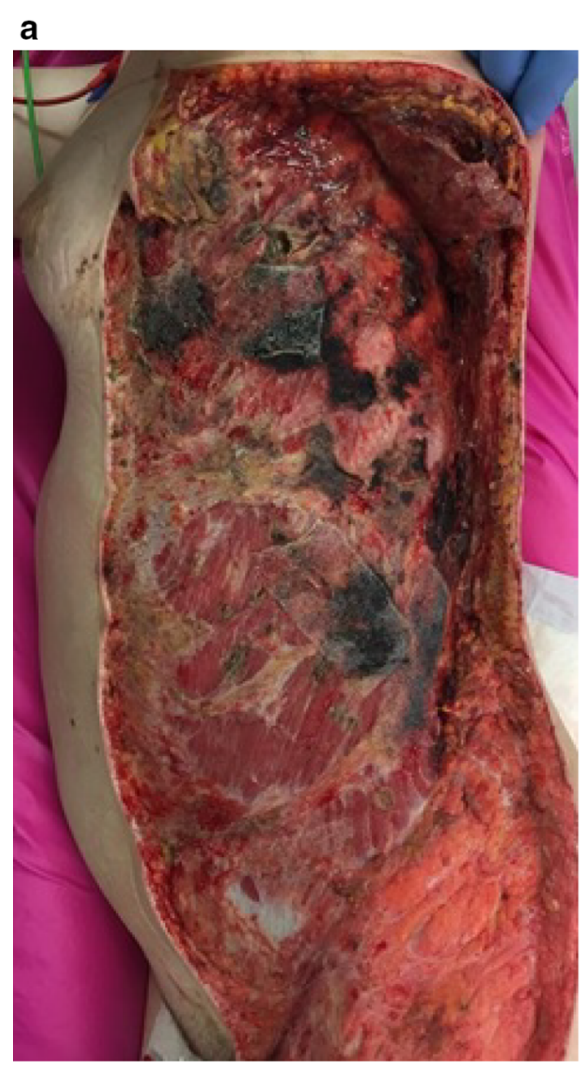

b

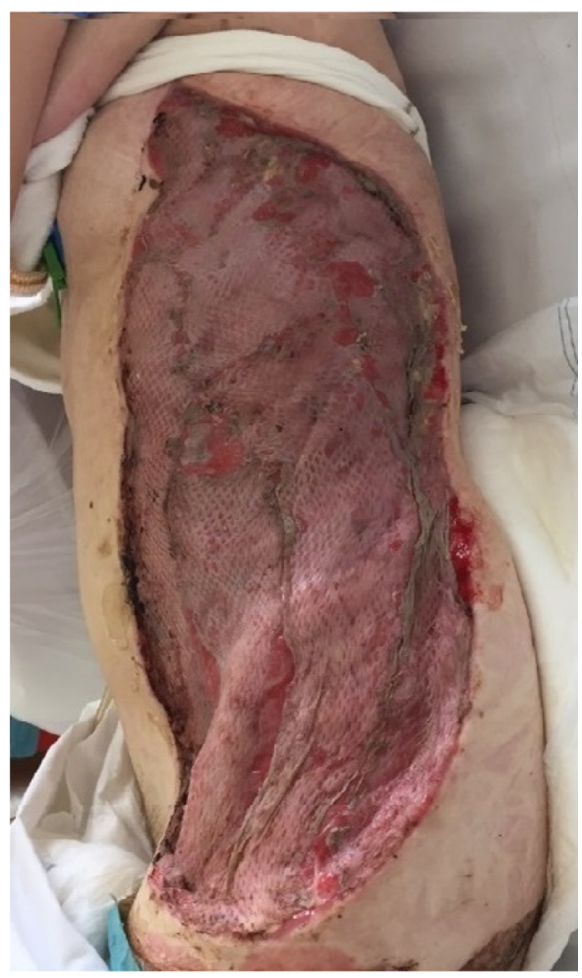

c

d
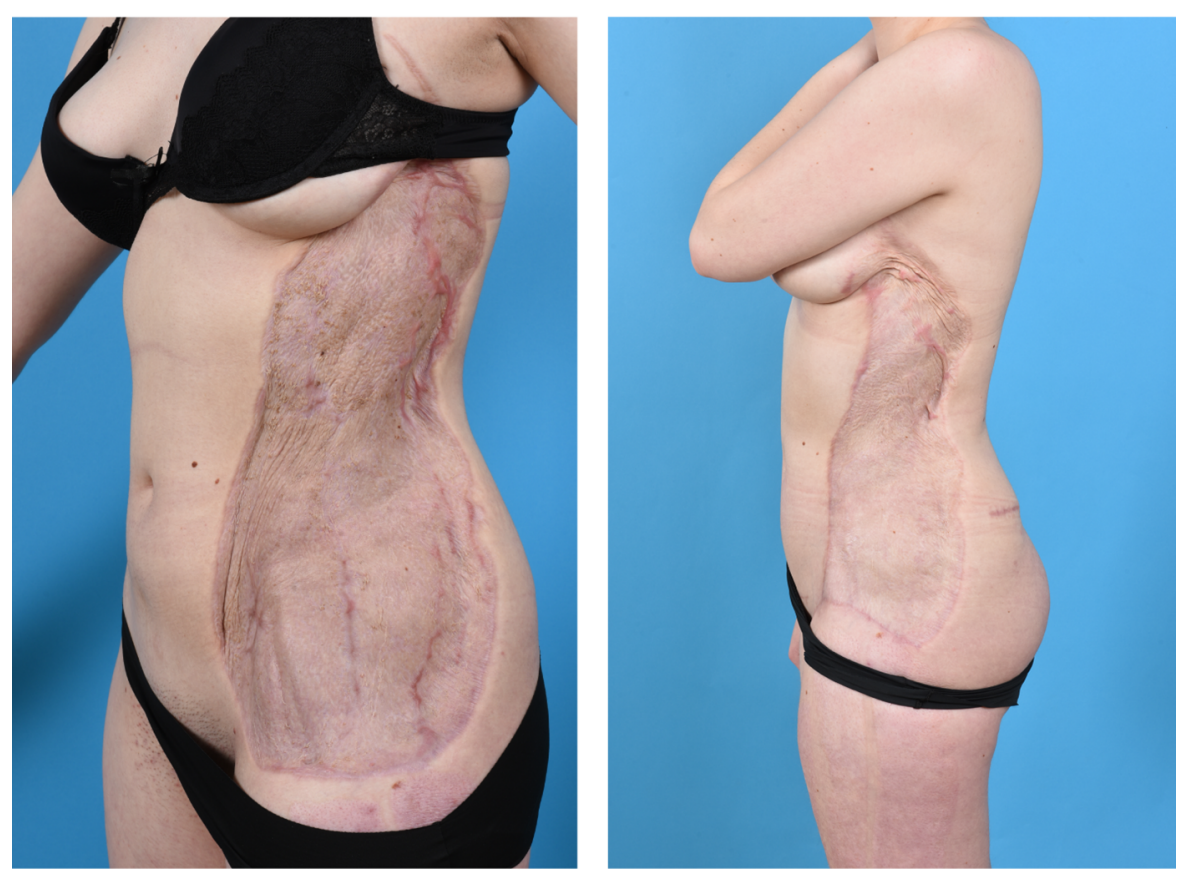
4Fig. 1 Case 1. a Before wound coverage (preparation of the wound bed with NPWT); b 10 days after Glyaderm + STSG application; c 6 months after Glyaderm + STSG application; d 1.5 year after Glyaderm + STSG application (after skin expansion and partial scar resection medially)

maximum of 95 degrees' flexion was reached. Further follow-up is planned to decide whether additional reconstruction is required to improve knee mobility.

\section{Outcomes and follow-up}

Ten patients were treated with Glyaderm and STSG in a single-stage procedure between 2017 and 2019 (Table 1). Patients were aged 3 weeks to 76 years old. Indications were: burns $(n=4)$, traumatic wounds $(n=4)$ and skin defects after soft tissue infections $(\mathrm{n}=2)$. The treated skin surface varied from 1 to $16 \%$ TBSA. The smaller defects were on hands or overlying joints, were skin elasticity is of utmost importance for function. In seven cases with large wound surfaces or in wounds spanning highly mobile areas, NPWT was applied immediately after wound coverage. Follow-up varied from 4 months to 1.5 years. No complications occurred after surgery. The mean take rate was $98 \%$. Two cases showed mild hypertrophy at the scar edges and two patients required a later re-intervention to further improve the scar aesthetic appearance, amongst which our first case.

\section{Discussion}

In 2008, Glyaderm was developed as a cost-efficient alternative for human acellular dermal substitutes (Richters et al. 2008). The price for Glyaderm being $€ 4.60 / \mathrm{cm}^{2}$ in 2020 . The de-cellularization method with $\mathrm{NaOH}$ on glycerol preserves the donor elastin fibers well. These fibers are important for the ingrowth of host fibroblast and blood vessels and function as a "guide" for the fibroblasts in the turnover of donor collagen into host collagen, resulting in a more natural newly formed dermis with assumingly better elasticity (Pirayesh et al. 2015).

In a pilot study Pirayesh et al. (2015) a two-staged reconstruction with Glyaderm + STSG was compared to STSG alone. The mean Glyaderm + STSG take rate was $91.55 \%$ and mean STSG alone take rate was $96.67 \%$. Although there was no statistically significant difference in take rate, the authors found a better elasticity of scars in the Glyaderm group. Objective measurements of scar color and pigmentation were not statistically different between the two groups, as were the subjective scar scales (Pirayesh et al. 2015).

To our knowledge, Glyaderm has only been described in a two-staged procedure. In the first stage, a

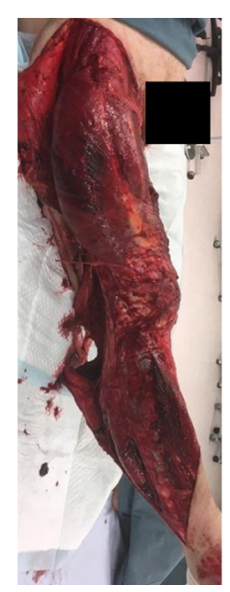

b

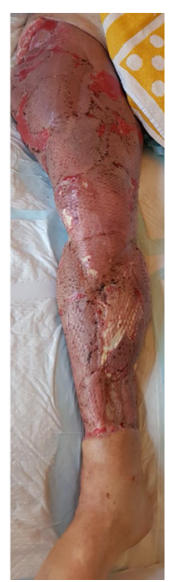

c

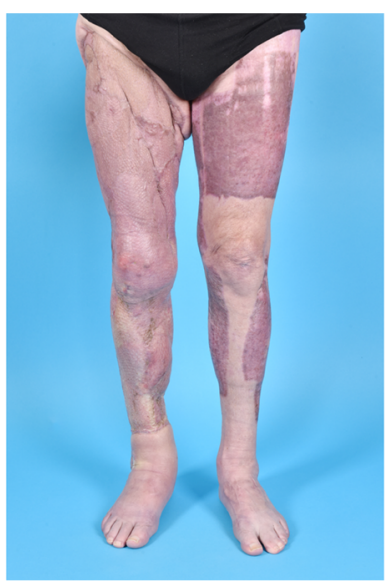

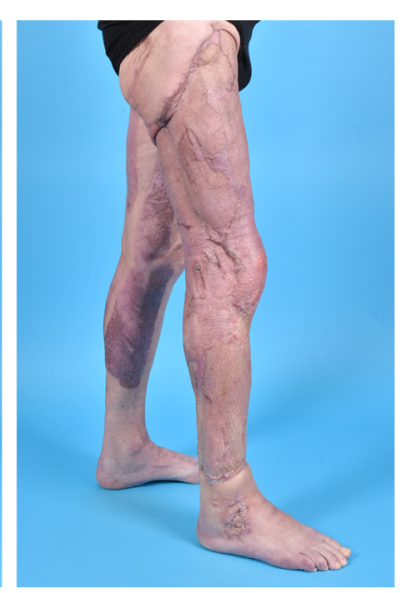

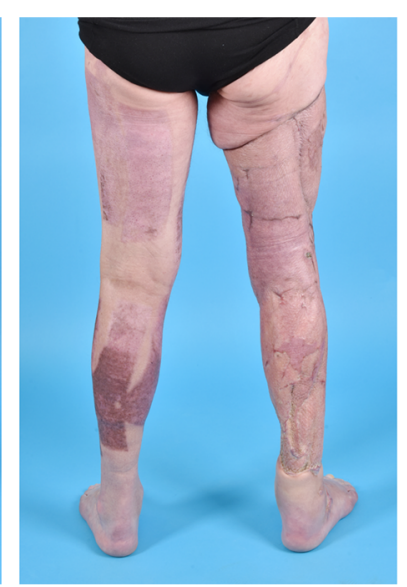

Fig. 2 Case 2. a Immediately after trauma; b 2 weeks after Glyaderm + STSG application; C 5 months after Glyaderm + STSG application 
Glyaderm is applied to a prepared wound bed, followed by the STSG autograft application 5-7 days later. Immediate application of Glyaderm to an unprepared wound bed was initially attempted, but showed a lack of ingrowth of Glyaderm, even after meshing it (Pirayesh et al. 2015). Since then, improvements have been made to the technical processing and adequate graft selection to obtain dermis' of uniform thickness, resulting in thinner and qualitatively more consistent dermal matrices. A thinner acellular dermal matrix improves vascular ingrowth possibilities from the granular wound bed to the STSG, providing it with oxygen and nutrients and increasing graft take. This article presents a case series were Glyaderm and STSG autografts were successfully applied in a single-staged procedure.

In this case series, some wounds were covered with a NPWT device after Glyaderm + STSG application. NPWT has been used since centuries to treat wounds (Miller 2012). NPWT in its most current form is used to heal complex wounds by draining excessive wound exudate and increasing the vascularity of the wound bed, resulting in increased granulation tissue formation compared to standard wound dressing (Argenta and Morykwas 1997; Sinha et al. 2013). Combining NPWT and dermal substitutes has already been studied in the past with good results (Molnar et al. 2004; Hutchison and Craw 2013). Liu et al. compared the use of acellular dermal matrix (ADM) with or without NPWT in treatment of deep burn wound in porcine limbs and found superior results in the ADM + NPWT group (Liu et al. 2016). Advantages of combining NPWT and dermal substitutes are less seroma formation, prevention of excessive movement during STSG ingrowth, easy wound care, good patient tolerance and consistent vascularization.

This is the first report describing the use of Glyaderm and STSG as a single-staged procedure, combined with or without NPWT treatment. Our cases show good results and suggest that bi-layered wound reconstruction with Glyaderm + STSG is feasible in a single-stage procedure. Our sample size is too small to draw definite conclusions and randomized controlled trials with larger patient numbers are needed to further assess results compared to STSG alone.

\section{Take home messages}

- An acellular dermal matrix serves as a collagen/ elastin scaffold to guide ingrowth of new dermal tissue

- Double-layered reconstruction might be recommended in areas overlying joints or in large wounds

- Glyaderm is of human origin and has lower costs compared to other biological ADMs

- A single-staged procedure for applying Glyaderm + STSG with or without NPWT is feasible and shows good clinical results in our first cases

- NPWT can be safely used in this procedure

Author contributions MDH: review of literature, writing of the manuscript. ASK: review of literature, acquisition and interpretation of data, writing and revising the manuscript. DJOU: conceptual design, acquisition of data, revising the manuscript and approval of the final version.

\section{Compliance with ethical standards}

Conflicts of interest A project in collaboration with Radboudumc and ETB- BISLIFE, Skin Department in Beverwijk (The Netherlands).

Open Access This article is licensed under a Creative Commons Attribution 4.0 International License, which permits use, sharing, adaptation, distribution and reproduction in any medium or format, as long as you give appropriate credit to the original author(s) and the source, provide a link to the Creative Commons licence, and indicate if changes were made. The images or other third party material in this article are included in the article's Creative Commons licence, unless indicated otherwise in a credit line to the material. If material is not included in the article's Creative Commons licence and your intended use is not permitted by statutory regulation or exceeds the permitted use, you will need to obtain permission directly from the copyright holder. To view a copy of this licence, visit http://creativecommons.org/licenses/by/4.0/.

\section{References}

Argenta LC, Morykwas MJ (1997) Vacuum-assisted closure: a new method for wound control and treatment: clinical experience. Ann Plast Surg 38(6):563-576

Butterfield JL (2013) 440 Consecutive immediate, implantbased, single-surgeon breast reconstructions in 281 patients: a comparison of early outcomes and costs between SurgiMend fetal bovine and AlloDerm human 
cadaveric acellular dermal matrices. Plast Reconstr Surg 131(5):940-951

Demling RH, Desanti L, Orgill DP (2007) Biosynthetic skin substitutes: purpose, properties and clinical indications, use of skin substitutes. http://www.burnsurgery.org

Hutchison RL, Craw JR (2013) Use of acellular dermal regeneration template combined with NPWT to treat complicated extremity wounds in children. J Wound Care 22(12):708-712

Lee KH (2000) Tissue-engineered human living skin substitutes: development and clinical application. Yonsei Med J 41(6):774-779

Liu W, Li F, Chen X, Pan Q (2016) Clinical efficacy of negativepressure wound therapy combined with porcine acellular dermal matrix for repairing deep burn wounds in limbs. Zhonghua Shao Shang Za Zhi 32(6):356-362

Miller C (2012) The history of negative pressure wound therapy (NPWT): from "Lip Service" to the modern vacuum system. J Am Coll Clin Wound Spec 4(3):61-62

Molnar JA, DeFranzo AJ, Hadaegh A, Morykwas MJ, Shen P, Argenta LC (2004) Acceleration of Integra incorporation in complex tissue defects with subatmospheric pressure. Plast Reconstr Surg 113(5):1339-1346

Nguyen DQ, Potokar TS, Price P (2010) An objective long-term evaluation of Integra (a dermal skin substitute) and split thickness skin grafts, in acute burns and reconstructive surgery. Burns 36(1):23-28

Pirayesh A, Monstrey S, Blondeel P, Hoekstra H, Vanoorbeek J, Hoeksema H, Richters CD (2007) Development of a novel dermal substitute based on glycerinized allograft: clinical (Phase I) and experimental evaluation. Burns 33:S13
Pirayesh A, Hoeksema H, Richters C, Verbelen J, Monstrey S (2015) Glyaderm((R)) dermal substitute: clinical application and long-term results in 55 patients. Burns 41(1):132-144

Richters CD, Pirayesh A, Hoeksema H, Kamperdijk EW, Kreis RW, Dutrieux RP, Monstrey S, Hoekstra MJ (2008) Development of a dermal matrix from glycerol preserved allogeneic skin. Cell Tissue Bank 9(4):309-315

Shahrokhi S, Arno A, Jeschke MG (2014) The use of dermal substitutes in burn surgery: acute phase. Wound Repair Regen 22(1): 14-22

Sinha K, Chauhan VD, Maheshwari R, Chauhan N, Rajan M, Agrawal A (2013) Vacuum assisted closure therapy versus standard wound therapy for open musculoskeletal injuries. Adv Orthop 2013:245940

Truong AT, Kowal-Vern A, Latenser BA, Wiley DE, Walter RJ (2005) Comparison of dermal substitutes in wound healing utilizing a nude mouse model. J Burns Wounds 4:e4

van der Veen VC, van der Wal MB, van Leeuwen MC, Ulrich MM, Middelkoop E (2010) Biological background of dermal substitutes. Burns 36(3):305-321

Wainwright DJ (1995) Use of an acellular allograft dermal matrix (AlloDerm) in the management of full-thickness burns. Burns 21(4):243-248

Publisher's Note Springer Nature remains neutral with regard to jurisdictional claims in published maps and institutional affiliations. 